\title{
Conformal Welding for Finitely Connected Regions
}

\author{
Donald E. Marshall
}

(Communicated by Thomas K. DeLillo)

\begin{abstract}
We discuss a numerical implementation of conformal welding for finitely connected regions using the geodesic zipper algorithm and Koebe's iterative method for computing conformal maps to regions bounded by circles. We also show that a conformal map from a finitely connected region to a region bounded by circles can be written as a composition of finitely many conformal maps of simply connected regions.
\end{abstract}

Keywords. Conformal welding, numerical conformal mapping, geodesic algorithm, zipper algorithm, Koebe's method.

2000 MSC. Primary 30C30; Secondary 30C35, 30C62.

\section{Introduction}

The Geodesic Algorithm, part of the zipper family of algorithms for the numerical computation of conformal maps, was originally developed in the 1980s to do conformal welding. There are several variants of conformal welding, all of which can be numerically approximated using the geodesic or zipper algorithms. One version is as follows: given a Jordan curve $J$, let $f$ and $g$ be conformal maps of the unit disk $\mathbb{D}=\{z:|z|<1\}$ and the exterior of the closed disk, $\mathbb{D}_{e}=\{z:|z|>1\}$, onto the two components of the complement of $J$. Then $h=f^{-1} \circ g$ is an increasing homeomorphism of the unit circle $\partial \mathbb{D}$ onto itself, called a welding homeomorphism. If we are given an increasing homeomorphism $h$ of $\partial \mathbb{D}$, the conformal welding problem is to find a Jordan curve $J$ and conformal (one-toone and analytic) maps $f$ and $g$ of the unit disk and its exterior onto the two components of the complement of $J$ so that $f(h(x))=g(x)$, for all $x \in \partial \mathbb{D}$. See Sharon and Mumford [8] for the application of conformal welding to vision or recognition problems. The welding homeomorphism $h$ is called the "fingerprint" of a region in their work. The welding is viewed by some authors as pasting together two hemispheres in a prescribed way on the bounding circles, with a

Received April 15, 2011, in revised form June 60, 2011.

Published online August 24, 2011.

Supported in part by NSF grant DMS-0900814.

ISSN 1617-9447/\$ 2.50 (C) 2011 Heldermann Verlag 
map which is conformal in each hemisphere. The image of the pasted circles is a Jordan curve on the sphere. Stereographic projection is a conformal map of the sphere (minus the north pole) onto the plane, and the map $1 / g(1 / z)$ extends to be conformal in a neighborhood of the origin, so the two versions are equivalent.

In this article we consider an extension of these concepts to finitely connected regions. Section 2 discusses welding homeomophisms associated with a finitely connected region viewed as the complement of Jordan regions on the sphere, and then gives a technique for solving the welding problem in this context. Along the way, we prove a theorem that may be of independent interest: a finitely connected region with $n$ holes can be conformally mapped onto the complement of $n$ disks using only $n$ conformal maps of simply connected regions. In Section 3 we discuss one of the main ingredients for the numerical computation of a conformal welding Koebe's iterative method for conformally mapping a finitely connected region to the complement of a union of disks. In Section 4 we give an example of the numerical solution of a conformal welding problem and an example of the computation of weldings associated with a finitely connected region. The technique used for conformal mapping of simply connected regions is the geodesic zipper algorithm. The geodesic zipper algorithm (see [6]) was invented in the 1980s to create a Jordan arc from a given welding of the positive reals to the negative reals. A simple modification immediately gave weldings of the unit circle to itself or the real line to itself, along with the associated conformal maps to the complementary regions. For the convenience of the reader, the procedure is outlined in Section 4. Reversing this procedure gave a method of computing conformal maps from the disk or a half plane to the interior and exterior of a given Jordan curve along with their inverse maps.

\section{Conformally welding disks onto the sphere}

We begin by describing how conformal maps of simply connected regions may be used for finitely connected regions. Let $\mathbb{S}^{2}$ denote the unit sphere, or Riemann sphere, in $\mathbb{R}^{3}$. The plane $\mathbb{C}$ together with the point "at infinity" is conformally equivalent to $\mathbb{S}^{2}$ via stereographic projection. A disk on the sphere will mean the conformal image of a disk via stereographic projection. The bounding circle is the intersection of a plane with $\mathbb{S}^{2}$. A (closed) Jordan curve on $\mathbb{S}^{2}$ determines two simply connected regions by the Jordan Curve Theorem, each of which is topologically equivalent to a disk. So by the Riemann Mapping Theorem and Koebe's Theorem [4, given $n$ disjoint simply connected regions $U_{j}$ on $\mathbb{S}^{2}$ bounded by disjoint Jordan curves, there exists conformal maps $g_{j}$ of $\mathbb{D}$ onto $U_{j}$ and a conformal map $f$ of $\mathbb{S}^{2} \backslash \bigcup_{j} D_{j}$ onto $\mathbb{S}^{2} \backslash \bigcup_{j} \overline{U_{j}}$ where $D_{j}$ are closed disks on $S^{2}$. Then $h_{j}=f^{-1} \circ g_{j}$ are called the welding homeomorphisms.

The conformal welding problem in this context is as follows: suppose $D_{1}, \ldots, D_{n}$ are $n$ closed disks on $\mathbb{S}^{2}$ and suppose that we are given $n$ homeomorphisms 
$h_{j}: \partial \mathbb{D} \rightarrow \partial D_{j}, j=1, \ldots, n$. Find conformal maps $g_{j}$ from $\mathbb{D}$ into $\mathbb{S}^{2}$ and a conformal map $f$ from $\mathbb{S}^{2} \backslash \bigcup_{j} D_{j}$ into $\mathbb{S}^{2}$ so that

$$
f\left(h_{j}(\zeta)\right)=g_{j}(\zeta)
$$

for all $\zeta \in \partial \mathbb{D}, j=1, \ldots, n$. More descriptively, the maps $g_{j}$ and $f$ conformally weld, or sew, copies of the unit disk into the holes $D_{j}$. The result is a sphere with patches, bounded by Jordan curves $J_{j}=g_{j}(\partial \mathbb{D})=f\left(\partial D_{j}\right)$.

Before proceeding, we mention some caveats to these procedures. The welding homeomorphism for a Jordan curve is not unique since each conformal map $g_{j}$ may be pre-composed with a conformal homeomorphism of the unit disk. This lack of uniqueness can be accounted for by a suitable normalization of the conformal maps. The Jordan curves obtained for a given homeomorphism also have a lack of uniqueness since we can post-compose with a Moebius map on the sphere. But again, this is avoided by a suitable normalization.

There are more serious impediments to this procedure if we allow arbitrary weldings and arbitrary Jordan curves. There are non-Moebius equivalent Jordan curves with the same welding and there are homeomorphisms which are not weldings. However these curves and homeomorphisms are extremely "wild" and not suitable for numerical calculation. For this reason we assume henceforth, unless stated otherwise, that all of our regions are bounded by quasicircles and that all welding homeomorphisms are quasisymmetric (see [3] for definitions of quasicircles and quasisymmetric). Then it is known that given an increasing quasisymmetric homeomorphism of the unit circle onto itself there is a quasiconformal conformal welding and each quasiconformal conformal welding uniquely determines (up to a Moebius map) a Jordan quasicircle in the plane. We point out the simple example of the welding $h\left(e^{i t}\right)=e^{i t / 2}$, when $0 \leq t \leq \pi$ and $h\left(e^{i t}\right)=e^{i(3 t / 2-\pi)}$, when $\pi \leq t \leq 2 \pi$ corresponds to a Jordan curve with an infinite spiral at the images of 1 and -1 . For numerical computations, we will restrict our attention to piecewise smooth curves and weldings so that they can be approximated by the discrete versions discussed below. The method also applies to quasicircles with small quasiconformal constants. Since any quasiconformal map can be written as a composition of quasiconformal maps with small constants, it can be applied as well to general quasicircles, but we do not treat this extension in this article.

For ease of description we will work on the plane instead of the sphere, keeping in mind that infinity corresponds to one point on the sphere. When convenient though, we will use the shorthand notation $\mathbb{S}^{2}$, as is common in this field. An increasing homeomorphism $h$ of the unit circle onto the circle $C=\{z:|z-c|=r\}$ is a homeomorphism $h: \partial \mathbb{D} \rightarrow C$ such that $\arg \left(h\left(e^{i \theta}\right)-c\right)$ is an increasing function of $\theta$. We require all homeomorphisms to be increasing for otherwise the welding conformal maps do not exist. We now describe how to solve the conformal welding problem for finitely connected regions. Suppose that we are given $n>1$ 
disjoint closed disks $D_{j} \subset \mathbb{C}$ and increasing homeomorphisms $h_{j}$, of the unit circle onto $\partial D_{j}, j=1, \ldots, n$. By the simply connected case, we can find a conformal map $f_{1}$ of the complement of $D_{1}, \mathbb{C} \backslash D_{1}$, into $\mathbb{C}$ and a conformal map $g_{1}$ of the unit disk into $\mathbb{C}$ so that each map extends continuously up to the bounding circle of their domains and such that $f_{1}\left(h_{1}(\zeta)\right)=g_{1}(\zeta)$ for all $\zeta \in \partial \mathbb{D}$. Set $\gamma_{1,1} \equiv g_{1}(\partial \mathbb{D})=f_{1}\left(\partial D_{1}\right)$. Next find a conformal map $k_{2}$ of the (punctured) simply connected region $\mathbb{C} \backslash f_{1}\left(D_{2}\right)$ onto the complement of a disk, $\mathbb{C} \backslash D_{2,2}$. The homeomorphism $h_{2}$ is then transplanted to

$$
H_{2}=k_{2} \circ f_{1} \circ h_{2}
$$

of $\partial \mathbb{D}$ onto $\partial D_{2,2}$.

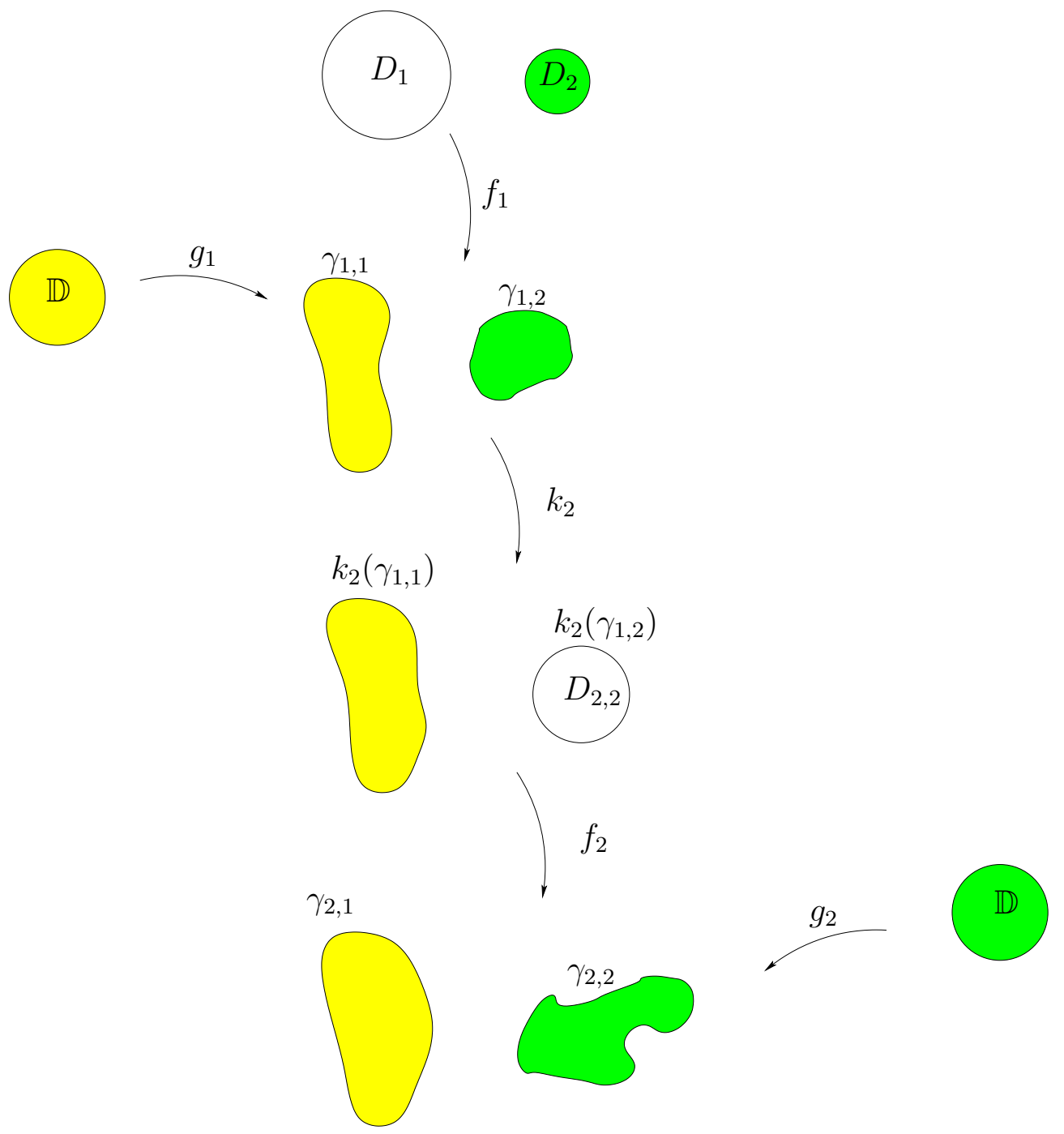

Figure 1. Welding two disks into the plane. 
The Jordan curve $\gamma_{1,1}$ is mapped to the Jordan curve $k_{2}\left(\gamma_{1,1}\right)$. We can now repeat this process by conformally welding the unit disk to $\partial D_{2,2}$ with the prescribed homeomorphism $H_{2}$ using conformal maps $f_{2}$ of $\mathbb{C} \backslash D_{2,2}$ and $g_{2}$ of $\mathbb{D}$ onto the complementary components of a Jordan curve $\gamma_{2,2}$. The image $\gamma_{2,1} \equiv f_{2}\left(k_{2}\left(\gamma_{1,1}\right)\right)$ remains a Jordan curve. Repeating this process for the successive images of the remaining disks yields the desired welding maps. The procedure uses $N$ weldings and $N-1$ additional conformal maps. See Figure 1 .

In the process of writing this paper, we realized that this approach is closely related to the earlier work of the physicist, B. Doyon [1, App. B] on certain doubly connected regions. Doyon proves that if a conformal function on a doubly connected region is sufficiently close to the identity map, then it can be factored as the composition of two functions defined on simply connected regions. $\mathrm{He}$ proves existence by analyzing certain integral equations for the factors. As can be seen from Figure 1, if we are given the doubly connected region at the bottom of Figure 1, then we can compose the map $k_{2}^{-1} \circ f_{2}^{-1}$ which is conformal on the region outside $\gamma_{2,2}$ with the map $f_{1}^{-1}$, which is analytic on the region outside $\gamma_{1,1}$ to obtain the map of the finitely connected region bounded $\gamma_{2,1} \cup \gamma_{2,2}$ onto the region bounded by $\partial D_{1} \cup \partial D_{2}$. If we find the welding homeomorphisms, using Koebe's Theorem for example, then we can apply the welding method described above to construct the desired maps. This idea also works when there are more than two boundary components, and there is no restriction on the boundaries.

Theorem 1 below follows then from the ideas given in Figure 1.

Theorem 1. Suppose $\Omega$ is a finitely connected region whose complement is a union of disjoint compact sets $K_{j}$, such that $\partial K_{j}$ is a quasicircle, $j=1, \ldots, n$. Then there are conformal maps $\varphi_{j}$ of simply connected regions $U_{j}$ into $\mathbb{C}$, for $j=1, \ldots, n$, so that

$$
\varphi=\varphi_{n} \circ \cdots \circ \varphi_{2} \circ \varphi_{1}
$$

is a conformal map of $\Omega$ onto a region bounded by $n$ circles. Moreover, each $\varphi_{j}$ has a quasiconformal extension to the sphere $\mathbb{S}^{2}$ so that (2) holds on $\mathbb{S}^{2}$. As a consequence, if $\Omega$ is any finitely connected region bounded by $n$ compact connected sets, then we can find $2 n$ conformal maps $\varphi_{j}$ of simply connected regions $U_{j}$, $j=1, \ldots, 2 n$ so that (2) holds with $n$ replaced by $2 n$.

Proof. See [3, Sec. VII.3] for background and references on quasicircles. First suppose that each $\partial K_{j}$ is a quasicircle. By Koebe's Theorem [4] we can find a conformal map $f$ of $\Omega$ onto the complement of $n$ disjoint closed disks $\left\{D_{j}\right\}$, and by the Riemann Mapping Theorem we can map each $K_{j}$ onto $\mathbb{D}$ with conformal maps $g_{j}$. By [5. Thm. II.8.3] the maps $g_{j}$ and $f$ have quasiconformal extensions to the sphere, since each boundary component is a quasicircle. As described earlier, $h_{j}=f \circ g_{j}^{-1}$ is a welding homeomorphism of the unit circle onto the $j^{\text {th }}$ circle $\partial D_{j}$ on the sphere, which must then be quasisymmetric. Armed with these weldings, we construct the inverses of the desired maps $\varphi_{j}$. First weld $\partial \mathbb{D}$ onto 
$\partial D_{n}$ using a conformal map $F_{n}$ of the complement of $D_{n}, \mathbb{S}^{2} \backslash D_{n}$, and a conformal map $G_{n}$ defined on $\mathbb{D}$ so that $h_{n}=F_{n}^{-1} \circ G_{n}$. The image $U_{n}=F_{n}\left(\mathbb{S}^{2} \backslash D_{n}\right)$ is a simply connected region. Then find a conformal map $L_{n-1}$ of the complement of the image of $F_{n}\left(D_{n-1}\right)$ onto the complement of a disk, $D_{n-1}^{\prime}$. Next weld $\partial \mathbb{D}$ onto $\partial D_{n-1}^{\prime}$ using a conformal map $M_{n-1}$ of $\mathbb{S}^{2} \backslash D_{n-1}^{\prime}$ and a conformal map $G_{n-1}$ defined on $\mathbb{D}$ so that

$$
L_{n-1} \circ F_{n} \circ h_{n-1}=M_{n-1}^{-1} \circ G_{n-1} .
$$

The map $F_{n-1}=M_{n-1} \circ L_{n-1}$ is a conformal map of the complement of $F_{n}\left(D_{n-1}\right)$ onto a simply connected region $U_{n-1}$. Repeat this process with the remaining boundary components. Then set $\varphi_{j}=F_{j}^{-1}$, and

$$
\varphi=\varphi_{n} \circ \cdots \circ \varphi_{2} \circ \varphi_{1}
$$

Then $\varphi$ is a conformal map of a finitely connected region $\varphi^{-1}\left(\mathbb{S}^{2} \backslash \cup D_{j}\right)$ onto $\mathbb{S}^{2} \backslash \cup D_{j}$. Note that

$$
F=F_{1} \circ F_{2} \circ \cdots \circ F_{n} \circ f
$$

is a conformal map of $\Omega$ into the plane. Likewise

$$
F_{1} \circ F_{2} \circ \cdots \circ F_{j-1} \circ G_{j} \circ g_{j}
$$

is a conformal map of the $j^{\text {th }}$ component of the complement of $\Omega$ onto the $j^{\text {th }}$ component of the complement of $\varphi^{-1}\left(\mathbb{S}^{2} \backslash \cup D_{j}\right)$ which agrees with $F$ on the boundary of the $j^{\text {th }}$ component of $\partial \Omega$ since the weldings are the same. We repeat this observation for each boundary component and hence obtain a homeomorphism of the extended plane or $\mathbb{S}^{2}$ onto itself which is conformal off finitely many quasicircles. But quasicircles are removable for homeomorphims which are conformal off the quasicircles and so we obtain a conformal map of the sphere onto itself, which must then be a Moebius map. Thus $f=\varphi \circ M$ where $M$ is a Moebius map of the sphere. Replacing $\varphi_{1}$ by $\varphi_{1} \circ M$ we obtain a factorization of $f$ as a composition of $n$ conformal maps of simply connected regions. By 3. Cor. VII.3.4], each $\varphi_{j}$ has an extension to a quasiconformal homeomorphism of $\mathbb{C}$ onto $\mathbb{C}$ or $\mathbb{S}^{2}$ onto $\mathbb{S}^{2}$.

Now suppose that $\Omega$ is any finitely connected region bounded by compact sets $K_{1}, \ldots, K_{n}$. By applying a Moebius map if necessary, we may suppose infinity is an interior point of $\Omega$. There exists a conformal map $\psi_{1}$ of the unbounded component of $\mathbb{C} \backslash K_{1}$ onto the complement of a disk $D_{1}$ by the Riemann Mapping Theorem. Again by the Riemann Mapping Theorem, there exists a conformal map $\psi_{2}$ of the unbounded component of $\mathbb{C} \backslash \psi_{1}\left(K_{2}\right)$ onto a disk. The image $\psi_{2}\left(\partial D_{1}\right)$ is an analytic curve. Repeating this process for each boundary curve we obtain a composition

$$
\psi_{n} \circ \cdots \circ \psi_{2} \circ \psi_{1}: \Omega \rightarrow \Omega^{\prime}
$$

where $\Omega^{\prime}$ is a region bounded by $n$ analytic curves. Now apply the first part of the proof to obtain the remaining $n$ conformal maps. 
It would be interesting if there were a way to construct these maps $\varphi_{j}$ directly, without first constructing the map $f$. After this article was refereed, we received a new preprint from B. Doyon proving that any conformal map (not just maps to regions bounded by circles) on any finitely connected domain can be factored as a composition of $n$ conformal maps of simply connected domains. Doyon uses the Uniformization Theorem in a clever way, instead of conformal welding.

\section{Koebe's method}

Given $n$ disjoint simply connected regions in the plane, we can compute the associated welding homeomorphism provided we have a method of computing a conformal map of the complement of (the closure of) these regions onto the complement of $n$ disjoint disks. In 1910, Koebe [4 proved that if $\Omega$ is a region whose complement consists of $n$ disjoint compact connected sets then there is a conformal map of $\Omega$ onto a region bounded by $n$ circles. He also outlined an iterative method for constructing such maps using only conformal maps of simply connected regions, though without a proof of convergence. In 1959, D. Gaier 2 proved that Koebe's iterative method converges. In fact it converges remarkably fast as we will illustrate.

Here is Koebe's method. Suppose $K_{1}, \ldots, K_{n}$ are disjoint compact connected sets in the plane. Let $\Omega=\mathbb{C} \backslash \bigcup_{j} K_{j}$. We assume that $\mathbb{C} \backslash K_{j}$ is connected, by filling in the holes in $K_{j}$ if necessary. Thus $\Omega$ is connected.

Find a conformal map $f_{1}$ of $\mathbb{C} \backslash K_{1}$ onto $\mathbb{C} \backslash D_{1}$, where $D_{1}$ is a closed disk. Then find a conformal map $f_{2}$ of $\mathbb{C} \backslash f_{1}\left(K_{2}\right)$ onto $\mathbb{C} \backslash D_{2}$ where $D_{2}$ is a closed disk. The image $f_{2}\left(D_{1}\right)$ is not a disk, but it is fairly close to a disk. Next find a conformal map $f_{3}$ of $\mathbb{C} \backslash f_{2} \circ f_{1}\left(K_{3}\right)$ onto $\mathbb{C} \backslash D_{3}$ where $D_{3}$ is a closed disk. Repeat the construction on the images in such a way that the images of each of the original compact sets are used infinitely many times.

To illustrate Koebe's method, consider the triply connected region in Figure 2(a) which is the complement of three compact sets: a star, a boomerang, and an anchor. The conformal maps below are computed using the geodesic zipper algorithm with about 500 boundary points. First we map the complement of the star to the complement of a disk. We normalize the map so that

$$
f_{1}(z)=z+\mathcal{O}(1 / z)
$$

by requiring that the image of infinity is infinity, and then applying a linear map if necessary. The result using the geodesic algorithm is given in Figure 2(b). Notice that the boomerang and anchor have hardly changed. Now compute the (normalized) conformal map of the complement of the image of the boomerang onto the complement of a disk. The result is given in Figure 2(c).

Notice that the disk which was the image of a star has image that is still almost a disk. Thirdly, find a conformal map of the second image of the anchor to 


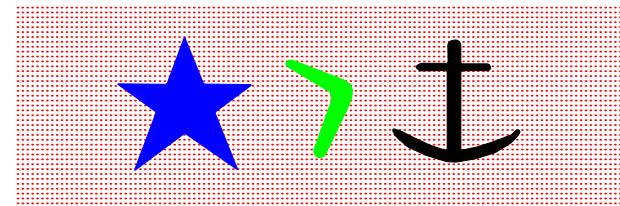

(a)

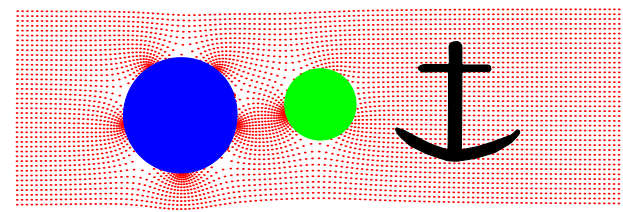

(c)

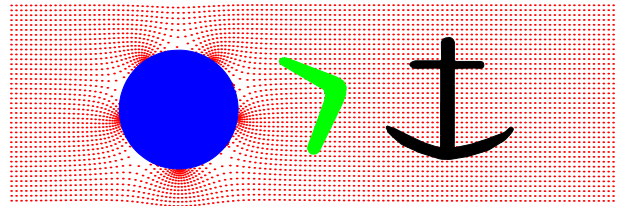

(b)

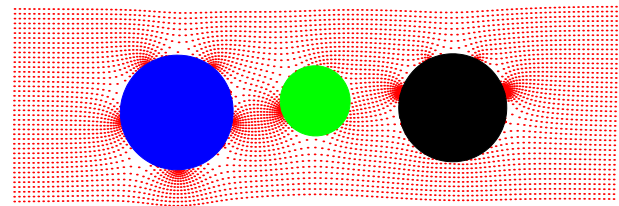

(d)

FiguRE 2. Koebe's method on a triply connected region.

the complement of a disk. See Figure 2(d). It is difficult after just these three maps to visually distinguish the resulting image from the complement of three disks. Koebe's method continues by choosing another one of the three compact complementary components of the region and mapping its complement to the complement of a disk, cycling through each of the three choices infinitely many times. Because of the limited resolution of postscript pictures and the compression algorithm used in conversion to pdf format, some artifacts might appear in printed or in electronic forms of these pictures which are not really present in the data. Higher resolutions versions are available upon request.

Of course these compact sets are rather far apart from a conformal mapping point of view. Figure 3 illustrates the application of Koebe's method (via the geodesic algorithm) in a more complicated situation. A Brownian traveler beginning at $\infty$ is extremely unlikely to hit the letters $R$ or $M$ before hitting $C$. This implies that the conformal images of the latter two letters must be very small compared to the image of the letter $C$.

- Figure 3(a) is the original region.

- Figure 3 (b) is the result after mapping the complement of the letter $\mathrm{C}$ to the complement of a disk.

- Figure3(c) is the same as 3(b) except magnified near the small components.

- Figure 3(d) is the result after mapping Figure 3(b) so that the letter R component becomes a disk. 
- Figure 3)(e) is the same as Figure 3(d) except magnified near the small components.

- Figure 3(f) is the result after mapping Figure 3(d) so that the letter M component becomes a disk.

- Figure 3(g) is the same as Figure 3(f) except magnified near the small components.

- Figure 3(h) is the result after applying three more conformal maps.

- Figure 3(i) is the same as Figure 3(h) except magnified near the small components.

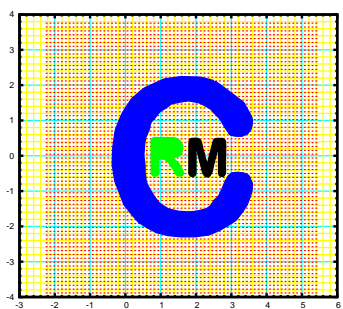

(a)

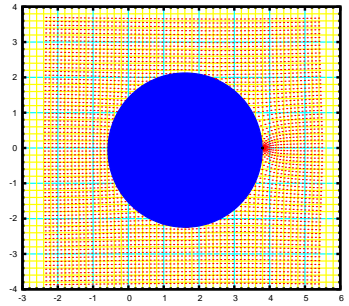

(d)

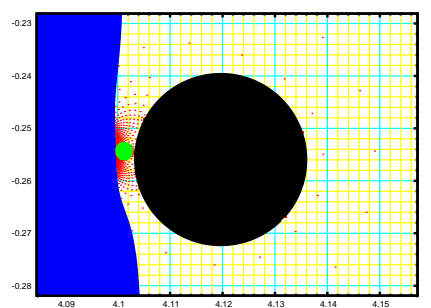

(g)

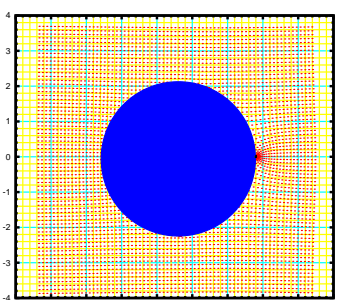

(b)

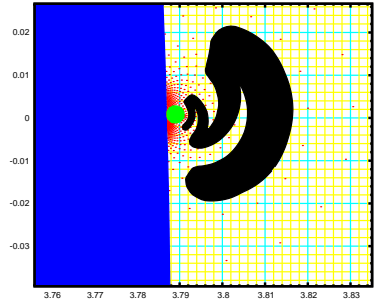

(e)

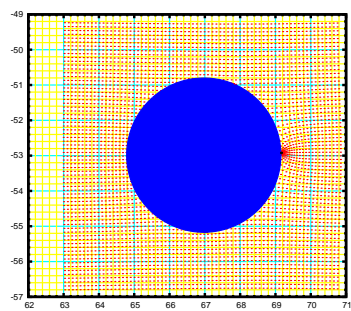

(h)

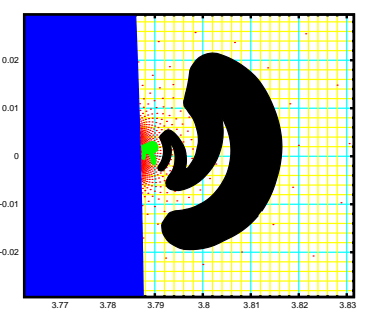

(c)

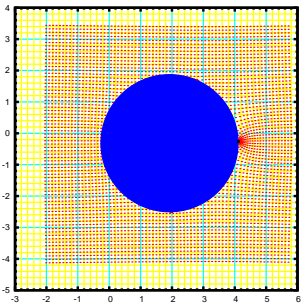

(f)

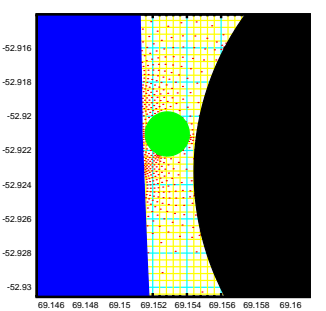

(i)

FiguRE 3. Koebe's method on a triply connected region.

Table 1 gives one method of comparing the images to a union of disks. The geodesic algorithm with about 500 points on each boundary was used in Figure 2 and about 1000 points on each boundary in Figure 3. Roundness of a boundary component was measured by taking an interior point of each component then finding the ratio of the radius of the smallest disk centered at that point which contains the bounded region over the radius of the largest disk centered at that 
point contained in the bounded region. No effort was made to choose optimal centers. To maintain an interior point, or "center" in each region, we simply normalized each interior map from the geodesic algorithm to send a given interior point to the center of the corresponding circle, and computed the images of the other "centers" by the map. Notice that the ratio is within $10 \%$ after just the first three conformal maps described above for the star, boomerang, anchor region, and has 7 correct digits after 9 maps. It takes longer, but not much longer for the CRM region.

\begin{tabular}{c|ccc} 
iteration & star & boomerang & anchor \\
\hline 3 & 1.0939577 & 1.0569160 & 1.0000000 \\
4 & 1.0000000 & 1.0558549 & 1.0003865 \\
5 & 1.0004536 & 1.0000000 & 1.0008445 \\
6 & 1.0004524 & 1.0000213 & 1.0000000 \\
7 & 1.0000000 & 1.0000160 & 1.0000015 \\
8 & 1.0000003 & 1.0000000 & 1.0000014
\end{tabular}

\begin{tabular}{c|ccc} 
iteration & $\mathrm{C}$ & $\mathrm{R}$ & $\mathrm{M}$ \\
\hline 3 & 1.0039654 & 1.3589302 & 1.0000000 \\
6 & 1.0002746 & 1.0206082 & 1.0000000 \\
9 & 1.0000260 & 1.0026309 & 1.0000000 \\
12 & 1.0000032 & 1.0002989 & 1.0000000 \\
15 & 1.0000000 & 1.0001116 & 1.0000000 \\
18 & 1.0000003 & 1.0000366 & 1.0000000
\end{tabular}

TABLE 1. Ratio of max to min radius about images of center points.

One of the advantages of the geodesic zipper algorithm is that it is fast and flexible enough to apply to any of these regions. Each map above takes less than a couple of seconds to compute.

\section{Numerical solution of the welding problem}

To implement the solution to a finitely connected conformal welding problem, from the discussion in Section 2, we need a numerical method for welding the inside of a disk to the outside of a disk. There are several classical methods in the literature for solving this welding problem theoretically. The "sewing method" in [5] involves repeated reflections, the PDE method in 7] involves solving a Beltrami equation. See $[8]$ for another method with a numerical implementation, albeit complicated. The zipper algorithm was invented in the 1980s as a simple, fast and accurate method to solve this welding problem numerically. 
For the convenience of the reader, we give a brief description of welding using the geodesic version of the zipper algorithm. For more details see [6]. Using linear maps of the plane, we may suppose that both disks are the unit disk. A welding map $h\left(e^{i \theta}\right)$ of the unit circle to itself, which after a rotation will satisfy $h(-1)=-1$, can be easily illustrated if it is given in the form $(\theta, w(\theta))$ where $w(\theta)=\arg \left(h\left(e^{i \theta}\right)\right)$, an increasing homeomorphism of $[-\pi, \pi]$ onto $[-\pi, \pi]$. We can approximate a given map $w$, for instance, by selecting a subdivision $-\pi=\theta_{0}<\theta_{1}<\theta_{2}<\ldots<\theta_{n}=\pi$. The zipper algorithm will create an increasing homeomorphism $w_{c}$ so that $w_{c}\left(\theta_{j}\right)=w\left(\theta_{j}\right), j=0, \ldots, n$. Since both maps are increasing homeomorphisms, we easily then have

$$
\max _{\theta}\left|w_{c}(\theta)-w(\theta)\right| \leq \max _{j}\left|w\left(\theta_{j}\right)-w\left(\theta_{j-1}\right)\right| .
$$

Let

$$
C(z)=i \frac{1-z}{1+z}
$$

be a linear fractional transformation which maps the unit disk to the upper half plane, the exterior of the disk to the lower half plane and the unit circle to the real line. Then

$$
h_{\mathbb{R}}=C \circ e^{i w} \circ C^{-1}
$$

is an increasing homeomorphism of the real line to itself. Set $x_{j}=C\left(e^{i \theta_{j}}\right)$ and $y_{j}=C\left(e^{i w\left(\theta_{j}\right)}\right)$. We seek conformal maps $f$ and $g$ of the upper and lower half plane onto the interior and exterior of a Jordan curve so that $f\left(x_{j}\right)=g\left(y_{j}\right)$, $j=0, \ldots, n$. In other words, the intervals $I_{j}=\left(x_{j-1}, x_{j}\right)$ and $J_{j}=\left(y_{j-1}, y_{j}\right)$ will be mapped to a common arc of the Jordan curve. First apply linear maps to the upper and lower half plane so that without loss of generality, $I_{n}=(0,+\infty)$ and $J_{n}=(0,+\infty)$. We view the points $x_{j}$ as lying on the boundary of the upper half plane and the points $y_{j}$ as lying on the boundary of the lower half plane. Apply the map $i \sqrt{z}$ to $\mathbb{C} \backslash(-\infty, 0]$, so that the points $x_{j}$ will be mapped to an increasing sequence of points in $(-\infty, 0]$ and the points $y_{j}$ will be mapped to a decreasing sequence of points in $[0, \infty)$. The image of $I_{n}$ and $J_{n}$, the positive reals, is the positive imaginary axis. The images of $I_{n-1}$ and $J_{n-1}$ are two intervals meeting at 0 . Apply a linear fractional transformation of the upper half plane onto itself so that the image of these two intervals become $[-1,0]$ and $[0,1]$. The map $\sqrt{z^{2}-1}$ is then a conformal map of the upper half plane $\mathbb{H}$ onto $\mathbb{H} \backslash I$, where $I$ is the line segment from 0 to $i$. Then $I$ is the image of the original intervals $I_{n-1}$ and $J_{n-1}$. The positive imaginary axis is mapped to a curve in $\mathbb{H}$ from $i$ to a point on the extended real line. The images of $I_{n-2}$ and $J_{n-2}$ are now two intervals meeting at 0 , and by a linear fractional transformation we can map them to $[-1,0]$ and $[0,1]$. Applying $\sqrt{z^{2}-1}$ again we "paste" the two intervals corresponding to $I_{n-2}$ and $J_{n-2}$ to obtain the interval $I$.

Repeat this construction until only the image of $I_{1}$ and $J_{1}$ remain on $\mathbb{R}$. Applying another linear fractional transformation, we may assume that the image 
of $I_{1}=(-\infty, 0]$ and the image of $J_{1}=[0, \infty)$. The image of all other (welded) intervals forms a curve in $\mathbb{H}$ from 0 to $\infty$. Now apply the map $z^{2}$. If we compose the initial linear maps $L_{1}$ and $L_{2}$ of the upper and lower half planes with this sequence of constructed maps we obtain conformal maps $f$ and $g$ of the upper and lower half plane onto the two components of the complement of a Jordan curve passing through infinity. If a bounded Jordan curve is desired, simply compose both maps with a linear fractional transformation. The inverse of each of these maps may be found by composing the inverses of each of their building blocks in the reverse order. Each inverse is either a linear fractional transformation or $\sqrt{z^{2}+1}$.

To give an example of the solution of a finitely connected conformal welding problem, we prescribe two disks and two welding maps. The welding maps $h_{j}\left(e^{i \theta}\right)$ are given in the form $\left(\theta, \arg \left(h_{j}\left(e^{i \theta}\right)-c_{j}\right)\right.$ as increasing homeomorphism of $[0,2 \pi]$ onto $[0,2 \pi]$, where $c_{j}$ is the center of the $j^{t h}$ disk, $j=1,2$. See Figure 4 The red (lower) and blue (upper) graphs correspond respectively to the red (left) and blue (right) circles.
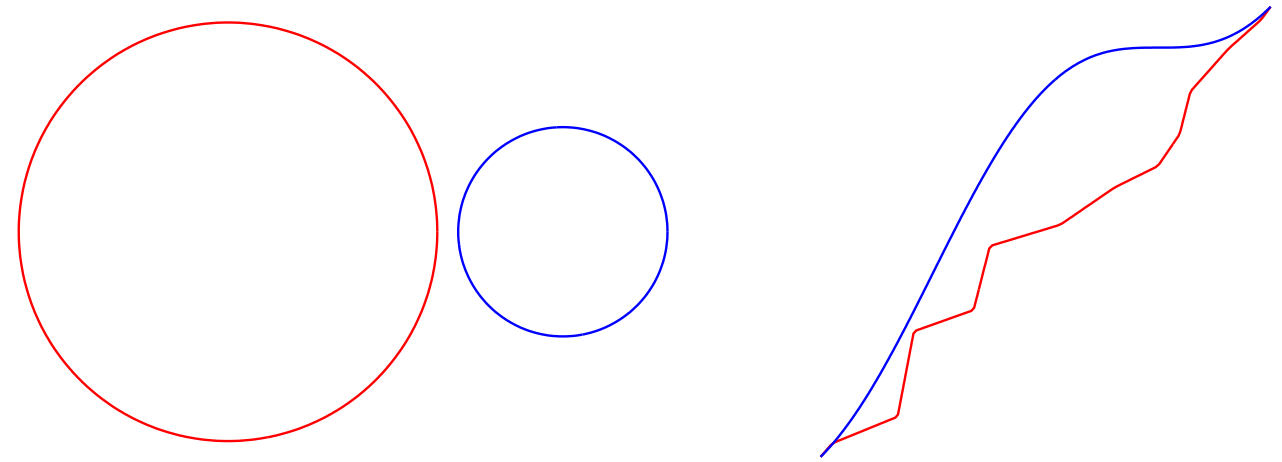

Figure 4. Two disks and two weldings maps.

Applying the process described in Section 2 (see Figure 11), using the zipper algorithm to create weldings, we obtain a doubly connected region $\Omega$ shown in Figure 5 (red on the left, blue on the right) along with a conformal map $f$ (expressed as a composition of conformal maps of simply connected regions) of the doubly connected region bounded by the two given disks $D_{j}$ onto $\Omega$ and conformal maps $g_{j}$ of the unit disk onto the interior of these two boundary curves so that the given weldings $w_{j}(\theta)$ satisfy

$$
\left.h_{j}\left(e^{i \theta}\right)=f^{-1} \circ g_{j}\left(e^{i \theta}\right)\right),
$$

where $w_{j}(\theta)=\arg \left(h_{j}\left(e^{i \theta}\right)-c_{j}\right)$. 


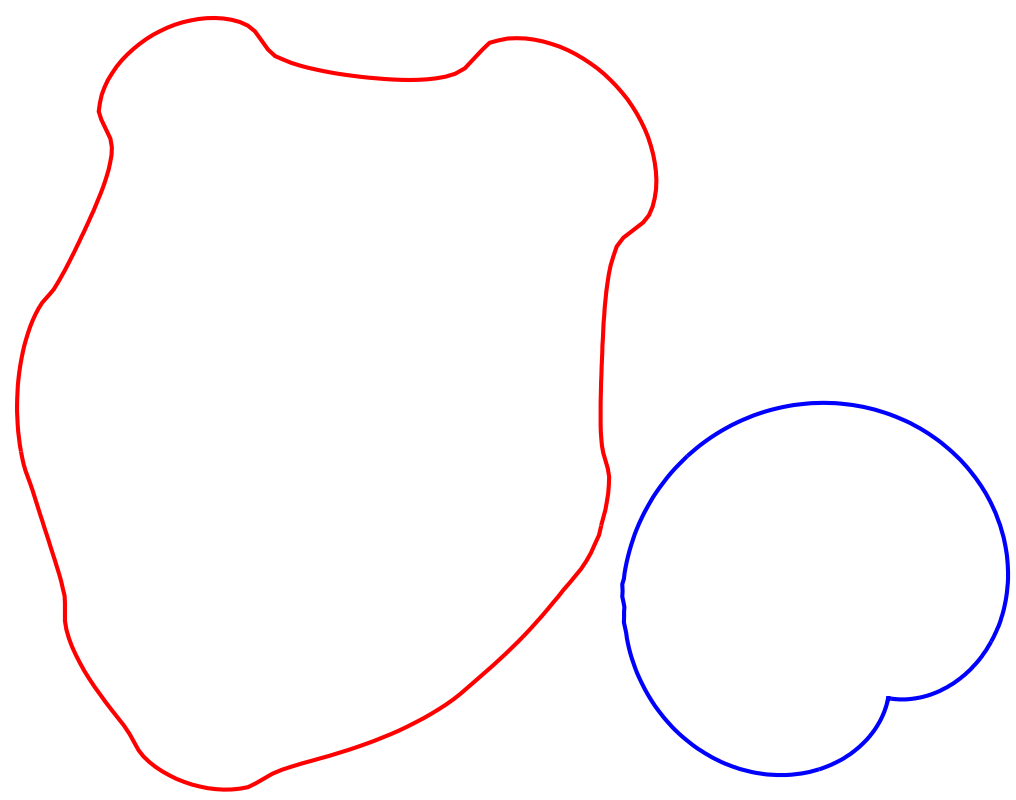

Figure 5. A welded doubly connected region.

We conclude with an example where we find the welding maps for a given finitely connected domain, namely the star-boomerang-anchor region of Figure 2. See Figure 6] As described in Section 2, we need the conformal map $f$ of this region (the region outside all three) to a region exterior to three disks and we need the conformal maps $g_{j}, j=1,2,3$, from the unit disk to the interior of the three regions. The weldings are then $h_{j}=f^{-1} \circ g_{j}$, which we display as graphs of increasing homeomorphisms of $[-\pi, \pi]$ as described earlier in this section. The geodesic zipper algorithm can be used to find these maps. A conformal map of the upper half plane to the interior of a Jordan curve and a conformal map of the lower half plane to the exterior of the Jordan curve are simultaneously computed by basically reversing the welding steps described above. At each step, the inverses are also available as elementary maps. See [6] for details. We use these maps at each stage of the Koebe algorithm described in Section 3 to obtain maps of the extended plane minus a Jordan curve to the extended plane minus a circle, and simply compose the sequence of maps. After a (rather small) finite number of iterations of Koebe's method using the geodesic zipper algorithm, we then obtain by composition a conformal map of the original star-boomerang-anchor region to the complement of three disks and conformal maps of the interiors of the star, boomerang, and anchor to the interior of three disks. The inverses of the latter maps are simultaneously found by the zipper algorithm. Composing the inverses gives the welding homeomorphisms.

See Figure 6 for the resulting welding homeomorphisms. 


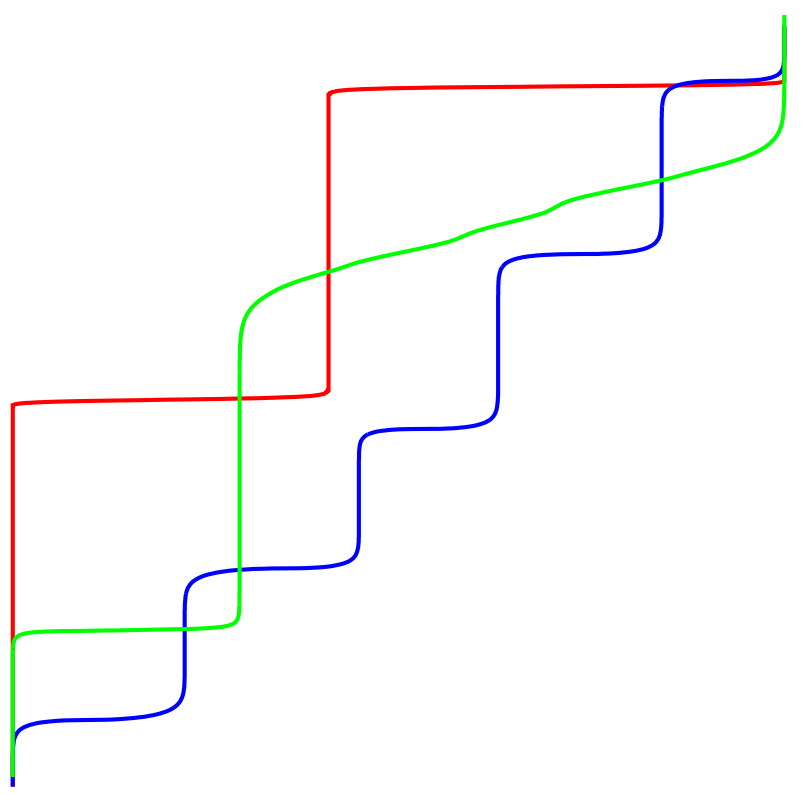

FiguRE 6. Welding homeomorphisms for the star-boomeranganchor region.

We leave it as an informative exercise for the reader to determine which of the three homeomorphisms corresponds to each boundary curve. As a hint, we suggest a careful study of Figure 2 and remind the reader of the highly non-Euclidean compression and expansion for boundary values of conformal maps.

Acknowledgement. The author expresses his thanks to the Centre de Recerca Matemàtica, Barcelona, for their hospitality while much of this work was completed.

\section{References}

1. B. Doyon, Calculus on manifolds of conformal maps and CFT, arXiv:1004.0138v2 [math$\mathrm{ph}$, 2010-06-15.

2. D. Gaier, Untersuchung zur Durchführung der konformen Abbildung mehrfach zusammenhängender Gebiete, Arch. Rat. Mech. Anal. 3 (1959), 149-178.

3. J. Garnett and D. E. Marshall, Harmonic Measure, Cambridge Univ. Press, 2005.

4. P. Koebe, Über die konforme Abbildung mehrfach-zusammenhängender Bereiche, Jahresber. Deut. Math. Ver. 19 (1910), 339-348.

5. O. Lehto and K. I. Virtanen, Quasicoformal Mappings in the Plane, Springer-Verlag, 1973.

6. D. E. Marshall and S. Rohde, Convergence of a variant of the Zipper algorithm for conformal mapping, SIAM J. Numer. Anal. 45 no.6 (2007), 2577-2609.

7. A. Pfluger, Über die Konstruktion Riemannscher Flächen durch Verheftung, J. Indian Math. Soc. 24 (1961), 401-412.

8. E. Sharon and D. Mumford, 2-D Shape analysis using conformal mapping, Int. J. Computer Vision 70 no.1 (2006), 55-75. 
AdDress: University of Washington, Department of Mathematics, Box 354350, Seattle, WA 98195-4350 U.S.A.

\title{
Addendum to Conformal Welding for Finitely Connected Regions
}

\author{
Donald E. Marshall
}

After the online version of this article first appeared, A. Solynin pointed out that Theorem 1] was announced in [A]. Proofs appeared subsequently in $[\mathrm{A}, \mathrm{A}, \mathrm{B}]$.

\section{References}

A1. V. Erohin, On the theory of conformal and quasiconformal mapping of multiply connected regions, Dokl. Akad. Nauk SSSR 127 (1959), 1155-1157 (in russian).

A2. O. Hübner, Funktionentheoretische Iterationsverfahren zur konformen Abbildung ein und mehrfach zusammenhängender Gebiete, Mitt. Math. Sem. Giessen 63 (1964).

A3. R. Kühnau, Einige elementare Bemerkungen zur Theorie der konformen und quasikonformen Abbildungen, Math. Nach. 45 (1970), 307-316. 